Article

\title{
Transmission Channel Analysis for Broadband Communication over Multiconductor UIC Cables Onboard Regional Trains
}

\author{
Sami Barmada ${ }^{1, *(\mathbb{D}}$, Mauro Tucci $^{1}{ }^{1}$ and Francesco Romano ${ }^{2}$ \\ DESTEC, University of Pisa, 56122 Pisa, Italy; mauro.tucci@unipi.it \\ 2 Titagarh Firema Adler S.p.A., 81100 Caserta, Italy; francesco.romano@titagarh.it \\ * Correspondence: sami.barmada@unipi.it; Tel.: +39-050-2217312
}

Received: 29 December 2018; Accepted: 31 January 2019; Published: 4 February 2019

check for updates

\begin{abstract}
In this contribution, the authors perform a detailed analysis relating to the use of the multiconductor UIC (Union Internationale des Chemins de fer) cables as a broadband data transmission channel onboard regional trains. The analysis is performed evaluating the channel frequency response and the channel capacity as a function of the distance between communication devices, in the 2-30 MHz frequency range of broadband powerline communications. The per unit length (p.u.l.) parameters are measured and simulated in a finite element method (FEM) code, allowing accurate full multiconductor simulations to evaluate the attenuations of the selected channel for communication and the cross-talk between cables. This study has been conducted considering that there are other signals carried by the cable, during regular train operation, and their integrity needs to be taken into account. The results show that, as a matter of fact, UIC cables can be used to transmit high-speed data; this can be exploited for introducing additional facilities (i.e., infotainment, safety features and so on) onboard regional trains, at a reasonably low cost, since no new cabling harnesses need be installed.
\end{abstract}

Keywords: data transmission; multiconductor cable; broadband communication; channel modeling; onboard communication

\section{Introduction}

Modern railway systems follow trends in the automotive market: The modern traveler expects to be offered services such as onboard entertainment, high-speed internet connection, detailed information about the trip and so on. In addition, nowadays, the increase in the need for onboard safety leads to the requirement of onboard cameras located in each hauled stock.

All this new equipment requires more dedicated cable networks but is characterized by the following drawback: New cables mean additional costs, weight and space (the latter ones being secondary problems in railways systems if compared to cars, airplanes or satellites). This holds for new trains, while for old trains (for instance, regional trains), the revamping procedure could be extremely expensive, basically making it impossible to be realized.

The use of a broadband power line (BPL, or high-speed power line communications, PLC) in vehicles is an application that has been investigated by many researchers lately (see, for instance, [1-13]), showing that this technology could be of great help in reducing the need for cable harnesses. The research for in-vehicle PLC is largely devoted to automotive and electric cars [1-8], and in part to other vehicles such as ships [8-11] and aircraft [11-13]. The use of PLC technology to communicate with high-speed data rates over the UIC cables was a novel proposal by the authors, and the literature on PLC onboard trains is scarce. 
In this context, this work presents a complete characterization of the channel, extending and completing previous work where the authors already performed a feasibility study and selected a potential candidate cable for carrying the broadband signal, mainly the UIC cable comprising the remote control and communication line ([14-17]).

The rationale behind this choice is the following:

1. This electrical connection is extremely common in hauled stock and it is unified by regulation [14].

2. It connects the whole length of the train and, for safety and functional reasons, particular care should be taken to avoid any possible disconnection.

3. Its use could lead to an easy procedure for revamping older trains at a reduced cost.

4. In $[15,16]$, the cable has been tested and its electrical parameters have been measured and simulated; in addition, some simulations relating to data transmission are shown.

5. In Barmada et al. [17], the authors evaluate the channel capacity (hence the maximum data rate available) as a function of the noise produced by the other signals carried by the cable. The above described cable is a multiconductor transmission line and the crosstalk phenomena can create undesired voltages and currents which might disrupt either the BPL signal or the other signals.

This paper is based upon Barmada et al. [15-17], but the current paper includes the following additional research: a full multiconductor formulation for the evaluation of the frequency response and the crosstalk is used and described in detail, and the obtained frequency response is analyzed in depth, evaluating the delay spread and the cumulative distribution function of the frequency response attenuations. Additional research carried out and discussed in this work includes an evaluation of the bit error rate (BER) using a QPSK-OFDM (quadrature phase shift keying-orthogonal frequency division multiplexing) modulation scheme, giving us an indication of the degradation of the BER with distance.

A detailed analysis of signal propagation is carried out in this work, taking into account the characteristics of all the signals carried by this cable during regular train operation, with the scope of assessing the real feasibility of such an application, ensuring that both new signals (used for example for infotainment) and standard signals carried by the cable do not interact with each other.

It is fundamental to underline that these results show the eventual theoretical feasibility of the BPL implementation, but a thorough experimental measurement campaign is needed to assess the simulation results and verify the practical feasibility.

\section{System Description}

\subsection{UIC Cable Descrcription}

As described in [14], most trains running on European tracks are equipped with UIC cables, being the main core of the remote control and communication line. In particular, an 18-conductor flexible shielded cable (terminated by a plug) connects the stocks to one another and is connected (inside each stock) to a connection box (present at both ends of each vehicle). Inside the vehicle, the 18 conductors are divided into two different cables: 16-conductor and two-conductor cables, both shielded. Figure 1 shows the section of the 16-conductor cable with the cable numbering as explained in [14].

The 16 conductors have different roles in the train control, and they are divided into functional groups: Each single group has one conductor serving as a reference and one or more conductors as signal line. A brief description of the role of the conductors is the following:

1. Conductors 1 to 8 are by default dedicated to the transmission of voice signals and are characterized by AC signals at a frequency range 100-8000 Hz, and permanent DC signals characterized by a value between 18 and $33 \mathrm{~V}$.

2. Conductors 9 to 16 are by default dedicated to the transmission of DC pulses (with amplitude between 15 and $33 \mathrm{~V}$ and 2-second duration) for doors opening and closing and for the light signals "stop" and "go", with conductor \#12 as common ground. 
3. In addition, some users (German railways, DB, Austrian railways, ÖBB, French railways, SNFC, and Swiss railways, CFF) utilize conductors 9 to 12 for additional functions.

4. Conductors 9 to 12 are dedicated to the emergency brake and the electropneumatic actuators (DC pulses with value between 15 and $33 \mathrm{~V}, \mathrm{DB}, \mathrm{ÖBB}, \mathrm{CFF}$ ).

5. Conductors 9 and 10 can carry an $\mathrm{AC}$ signal at $1 \mathrm{kHz}$ relating to the emergency brake information (DB, ÖBB, CFF).

6. Conductors 10 and 11 carry AC signals (around $100 \mathrm{kHz}, 600-950 \mathrm{mV}$ ) relating to traction commands (DB).

7. Conductors 7, 9,10 and 11 carry additional signals in case the train has to leave the station in the opposite direction of its arrival (6 V, frequency band 10-20 kHz (SNCF).

8. Conductors 7 and 8 are used for voice messages in case there are external loudspeakers (DC signal, between 18 and $33 \mathrm{~V}, \mathrm{SNCF}$ ).

All the above conductors are good candidates to carry the broadband powerline channel, since standard OFDM (Orthogonal Frequency Division Multiplexing) protocols (such the widely diffused HomePlug AV) work in the frequency range $2-30 \mathrm{MHz}$ and there is no overlapping in the frequency bands mentioned above.

In $[15,16]$, the authors chose conductor \#12 as a return, and one of the conductors \#10 or \#11 (dedicated to the switching on and off of the light signals "stop" and "go") because of the specific default application ("stop" and "go" signal) with the application to the Italian national railway system.

Taking into account also the additional functions described above, the previous choice could still be used, but in this paper we investigate a different choice: the selected two-conductor line comprises conductors 5 and 6 . This pair of conductors is dedicated to the switching of the speakers and is characterized by DC signals of high magnitude (15-33 V), which are not affected by the low-power OFDM signal in a high-frequency band.

\subsection{UIC Cable Modelling and Measurements}

The first step to be performed when modeling a multiconductor transmission line is to obtain its per-unit length parameters. It is worth mentioning at this point that, due to the OFDM frequency range, it is of fundamental importance, in order to correctly evaluate the channel's performance, to characterize the frequency dependence of the parameters. For this reason, an accurate 2D Finite Element Method (FEM) model of the cable section (Figure 1) has been implemented, obtaining the R, $\mathrm{L}$ and $\mathrm{C}$ matrices. In this case, the frequency range typical of the OFDM protocol is between 2 and $30 \mathrm{MHz}$, and for this reason, the p.u.l. parameters have been calculated for three frequencies in the above-mentioned range, and the frequency behavior has been modeled as described in [17].

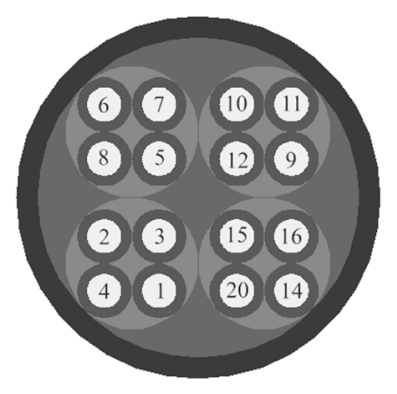

Figure 1. Section of the 16-conductor cable.

In order to validate the simulations, a measurement campaign has been carried out on a 6-m UIC cable. In particular, the measurements have been performed on a couple of conductors whose distances were minimum (i.e., $9-11$ or 14-16). The p.u.l. behavior in the range $0-30 \mathrm{MHz}$ is shown in Figures 2-5, showing good agreement with the parameters obtained by the FEM simulation. 
The measurement of the p.u.l. was performed using an Agilent Vector Network Analyzer and following the approach described in [18,19]. In particular, the approach described in [19] introduces a method to normalize the measurement with respect to the length of the cable, and the 6-m cable length is adequate to determine the p.u.l. in the frequency range of interest.

The relative error between measurements and simulations in the frequency range of interest is below $10 \%$. Some small divergences are caused by the measurement system, but the global frequency behavior of the measured p.u.l. parameters is well reproduced by the FEM model. While the p.u.l. L, $\mathrm{R}$ and $\mathrm{C}$ have been calculated and measured, the simple FEM model could not allow us to calculate the conductance (mainly because the dispersive properties of the insulating material composing the cable were not known); for this reason, the value of $G$ used in the channel simulations is taken directly from the measurements.

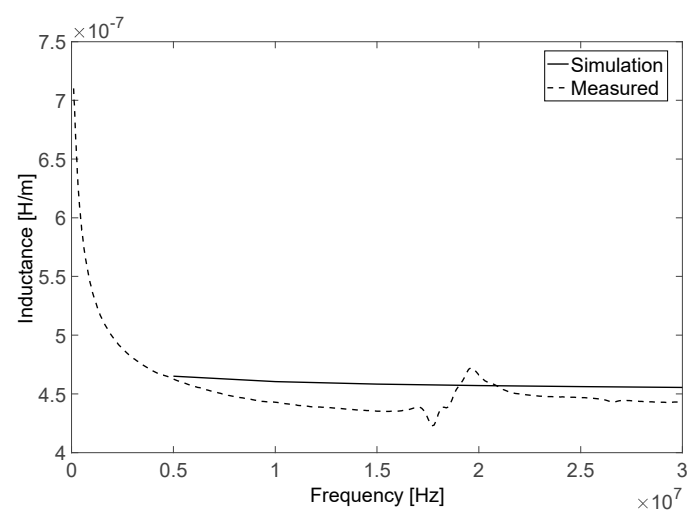

Figure 2. Measured and simulated inductance.

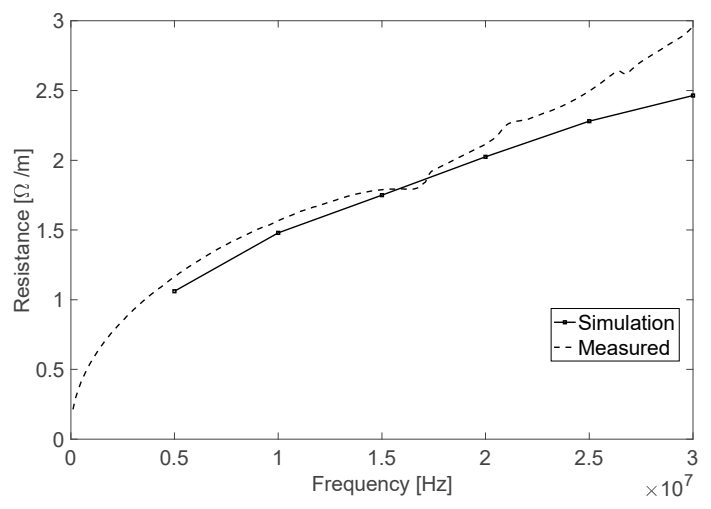

Figure 3. Measured and simulated resistance.

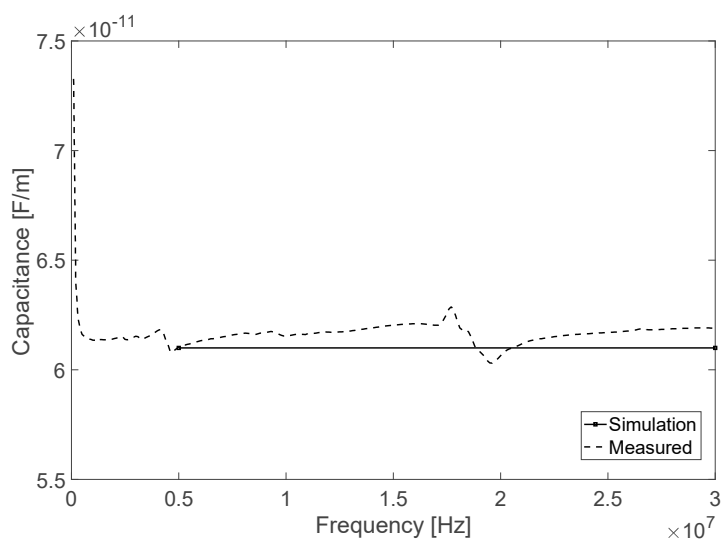

Figure 4. Measured capacitance. 


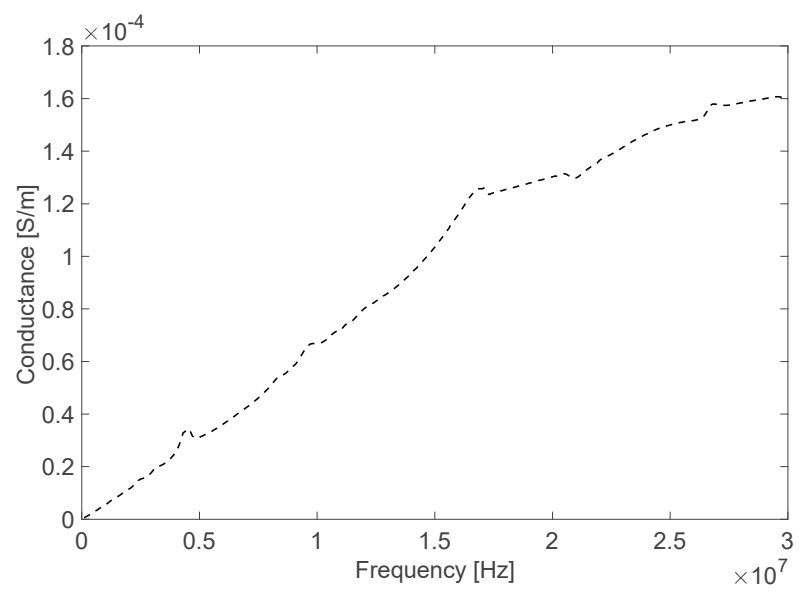

Figure 5. Measured conductance.

\subsection{Simulated System}

The whole system is a multiconductor transmission line, whose length is given by the number of hauled stocks composing the train. The electrical length of the cable inside each stock is about $L=20 \mathrm{~m}$; furthermore, $5 \mathrm{~m}$ can be considered the length of the connection between the two coaches. A model of seven hauled stocks has been considered (length $=175 \mathrm{~m}$ ) (see Figure 6).

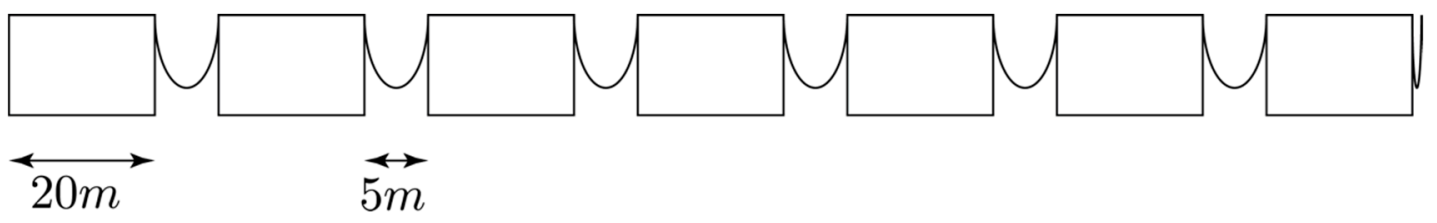

Figure 6. Outline of the simulation.

At the moment, the cable is terminated with an open circuit, but in order to optimize the communication performances, a different termination (typically the characteristic impedance) should be considered in theory.

Each single cable is dedicated to different functions and is closed on a termination impedance. Their values are described in [14], with the exception of conductors \#9 to \#15, in which a shunt resistance higher than $1200 \Omega$ is noted.

Each hauled stock should be equipped with a PLC modem capable of receiving/sending the PLC signal; these modems typically have a $50-\Omega$ resistance, and for this reason, in this paper, a $50-\Omega$ shunt resistance has been considered in each hauled stock. Any resistance of higher value, such as the ones mentioned before, has a negligible effect on the data transmission.

\subsection{Formulation of Signal Propagation over the Multiconductor Cable}

The model used for the simulation is based on the multiconductor transmission line (MTL) theory, since in the frequency range of interest the radiation phenomenon is still negligible if compared to conduction.

Figure 7 shows the equivalent circuit of a three-conductor transmission line relating to an elementary cell of length $\Delta z$ (the voltages are not shown for the sake of clarity in the picture).

The line model has been constructed in the frequency domain because the measured per-unit length parameters are characterized by frequency dependence and because at this stage, nonlinear behavior of the terminations is not taken into account.

The equations introduced from now on are relating to a three-conductor transmission line (as shown in Figure 7) in order to simplify the readability of the paper; however, the model relates to the full cable. 


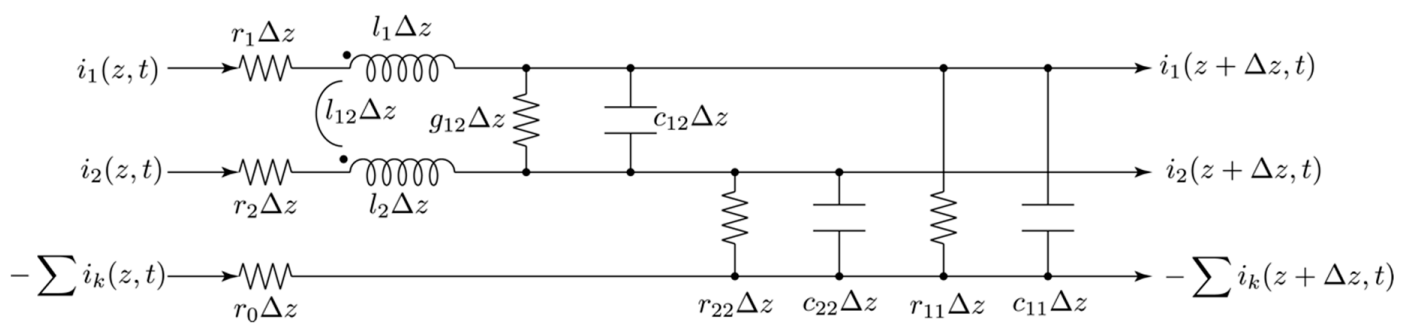

Figure 7. Multiconductor transmission line equivalent circuit.

In the frequency domain, the MTL equations are

$$
\left\{\begin{array}{c}
\frac{d \dot{V}(z)}{d z}=-\dot{r} \dot{I}(z)-j \omega \dot{I}(z)=-Z \dot{I}(z) \\
\frac{d \dot{I}(z)}{d z}=-g \dot{V}(z)-j \omega c \dot{V}(z)=-r \dot{V}(z)
\end{array},\right.
$$

which can be rewritten as

$$
\frac{d}{d z}\left[\begin{array}{c}
\dot{V}(z) \\
\dot{I}(z)
\end{array}\right]=-\left[\begin{array}{cc}
0 & \boldsymbol{Z} \\
\boldsymbol{Y} & 0
\end{array}\right]\left[\begin{array}{c}
\dot{V}(z) \\
\dot{I}(z)
\end{array}\right]=A\left[\begin{array}{c}
\dot{V}(z) \\
\dot{I}(z)
\end{array}\right],
$$

where $\dot{V}(z)=\left[\begin{array}{c}\dot{V}_{1}(z) \\ \dot{V}_{2}(z)\end{array}\right]$ and $\dot{I}(z)=\left[\begin{array}{c}\dot{I}_{1}(z) \\ \dot{I}_{2}(z)\end{array}\right]$, respectively, are the phasors of voltage and current at the beginning and at the end of the MTL cell, and the matrices relating to the parameters are:

$$
\begin{gathered}
\boldsymbol{r}=\left[\begin{array}{cc}
r_{1}+r_{0} & r_{0} \\
r_{0} & r_{2}+r_{0}
\end{array}\right], \boldsymbol{l}=\left[\begin{array}{ll}
l_{11} & l_{12} \\
l_{21} & l_{22}
\end{array}\right], \boldsymbol{c}=\left[\begin{array}{cc}
c_{11}+c_{12} & -c_{12} \\
-c_{21} & c_{21}+c_{22}
\end{array}\right], \\
\boldsymbol{g}=\left[\begin{array}{cc}
g_{11}+g_{12} & -g_{12} \\
-g_{21} & g_{21}+g_{22}
\end{array}\right] .
\end{gathered}
$$

The analytical solution for a single cell of length $L$ is consequently given by

$$
\left[\begin{array}{c}
\dot{V}(L) \\
\dot{I}(L)
\end{array}\right]=\exp (A L)\left[\begin{array}{c}
\dot{V}(0) \\
\dot{I}(0)
\end{array}\right]=\boldsymbol{K}\left[\begin{array}{c}
\dot{V}(0) \\
\dot{I}(0)
\end{array}\right],
$$

which basically corresponds to the inverse transmission parameter representation.

If $L$ is the length of a single stock, the previous equation coupled with the termination conditions (boundary equations) gives the voltage and current at the beginning and the end of each stock.

Considering that we are evaluating the behaviour of the system with respect to a PLC signal injected at termination \#1 (with a voltage generator), the boundary equations for a three-conductor MTL are the following:

$$
\left\{\begin{array}{c}
\dot{V}_{1}(0)=\dot{E}-R_{S 1} \dot{I}_{1}(0) \\
\dot{V}_{2}(0)=-R_{S 2} \dot{I}_{2}(0) \\
\dot{V}_{1}(L)=R_{L 1} \dot{I}_{1}(L) \\
\dot{V}_{2}(L)=-R_{L 2} \dot{I}_{2}(L)
\end{array}\right.
$$

where $R_{S 1}$ and $R_{S 2}$ are the termination resistances at the beginning of the line, while $R_{L 1}$ and $R_{L 2}$ are the termination resistances at the end of the line. At the beginning of each stock, a receiving termination (usually called a receiving modem) is considered. Its internal impedance can be considered (with a more than reasonable approximation) a resistance, and in this case, its value is fixed to $50 \Omega$. The equivalent cell of a modem (still relating to a three-conductor transmission line) is reported in Figure 8 , in which the modem resistance is connected between the active conductor and the return conductor; its representation in terms of inverse transmission parameters is given by: 


$$
\left[\begin{array}{c}
\dot{V}_{1 b} \\
\dot{V}_{2 b} \\
\dot{I}_{1 b} \\
\dot{I}_{2 b}
\end{array}\right]=\left[\begin{array}{cccc}
1 & 0 & 0 & 0 \\
0 & 1 & 0 & 0 \\
-1 / R_{m} & 0 & 1 & 0 \\
0 & 0 & 0 & 1
\end{array}\right]\left[\begin{array}{c}
\dot{V}_{1 a} \\
\dot{V}_{2 a} \\
\dot{I}_{1 a} \\
\dot{I}_{2 a}
\end{array}\right]=\boldsymbol{H}\left[\begin{array}{c}
\dot{V}_{1 a} \\
\dot{V}_{2 a} \\
\dot{I}_{1 a} \\
\dot{I}_{2 a}
\end{array}\right] .
$$

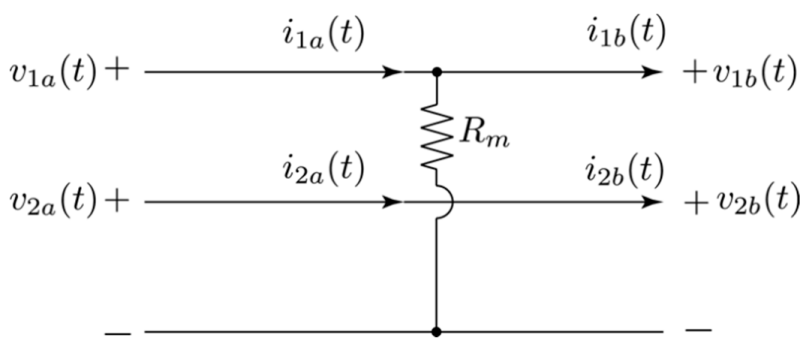

Figure 8. Elementary cell relating to the modem.

The previous equations can be used in two different ways: the first way is to use the cascade connection between the cells (stocks and modem), which allows the calculation of the quantities at the termination in an easy way by multiplying the matrices $\boldsymbol{K}$ and $\boldsymbol{H}$; the second way is to create a higher-dimension system leaving the voltages and currents at each modem as explicit unknowns. This second strategy leads to a higher-dimension system but allows a direct calculation of the transfer function between each modem (located in each stock) and the input voltage. For our purposes, the latter way is the suitable one: the following equation shows the final algebraic matrix relating to two stocks and a modem.

The $4 \times 4$ blocks whose entries are named $K$ relate to Equation (4), that is, the behaviour of each stock; the block whose entries are named $H$ relates to Equation (6), that is, the modem connection; and the other two rectangular blocks relate to the boundary conditions (terminations) as explained in Equation (5).

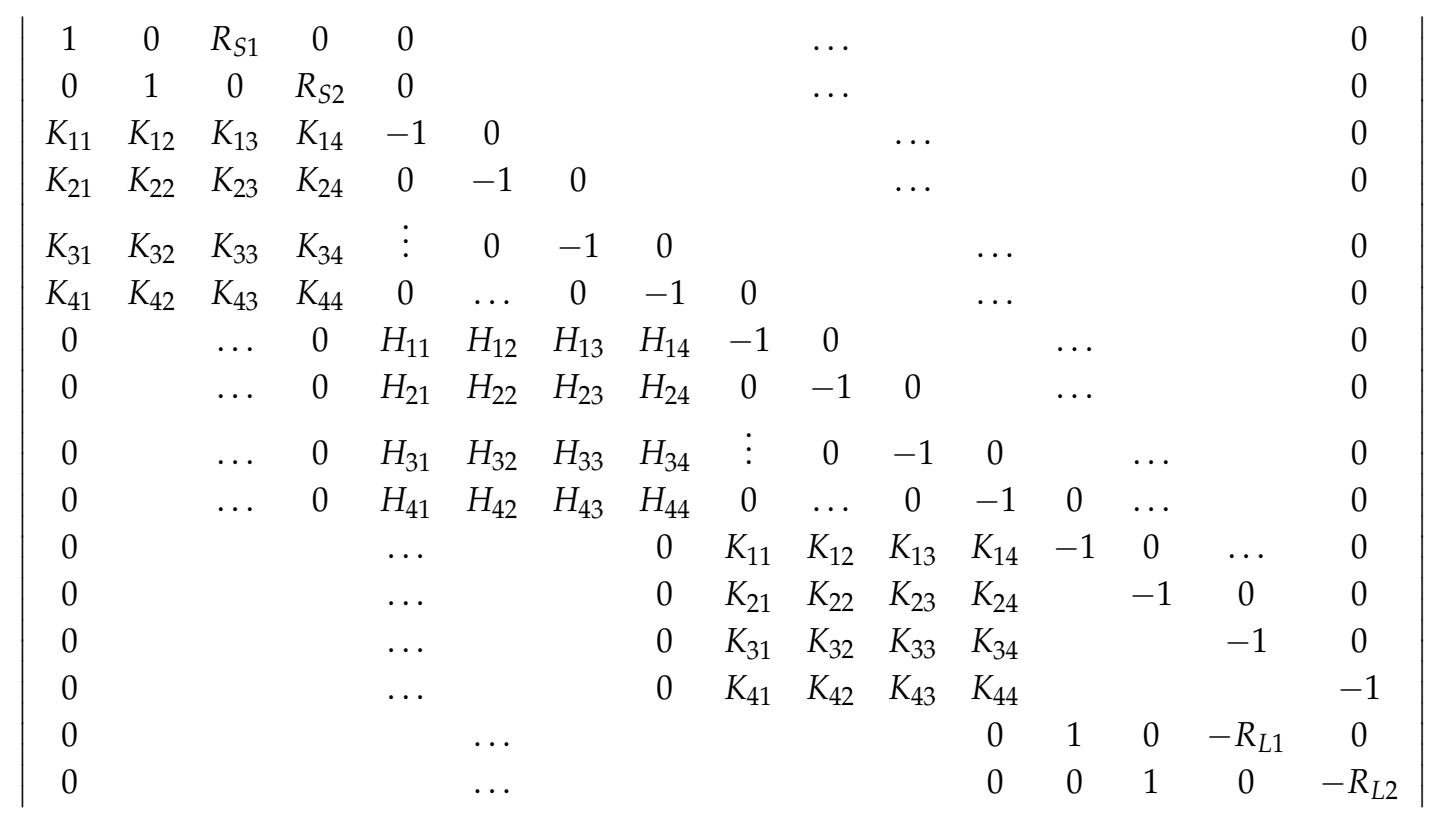

The algebraic system is solved for each frequency belonging to the range of interest; the voltage obtained at each modem termination is consequently used to calculate the transfer function relating to each receiver (the modem located in each stock) and the transmitter (the voltage generator).

In the next section, the results of the simulations are shown. 


\section{Simulation Results}

\subsection{Frequency Response and Crosstalk}

Figures 9 and 10 show the transfer function magnitude between the $n^{\text {th }}$ (with $n=1$ to 7 ) hauled stock and the first one (where the transmitter modem is supposed to be placed) in the frequency range $2-30 \mathrm{MHz}$. In particular, the transmitter modem impedance is (as before) considered as a $50-\Omega$ resistance, while the termination resistances of the conductors (the ones defined in Equation (5)) are set either equal to the characteristic impedance of the line (around $86 \Omega$ ) in Figure 9, or to an open circuit in Figure 10.

It can be observed that, as it is easy to predict, attenuation increases with distance from the modem and with frequency. Additional information that can be drawn from Figures 9 and 10 is the following: Considering a transmission power of $-55 \mathrm{dbm} / \mathrm{Hz}$ over the entire frequency band (a common value for PLC devices) and a background white Gaussian noise of $-80 \mathrm{dbm} / \mathrm{Hz}$ (which represents a highly noisy channel), this leads to a signal-to-noise power ratio at the transmitter of +25 db. PLC systems are designed to work even in noisy conditions near to $0 \mathrm{db}$ SNR at the receiver, so we obtain a maximum practicable attenuation of about $-30 \mathrm{db}$; for this reason, as can be seen from Figures 9 and 10, a repeater is needed every three hauled stocks if the whole frequency band is needed. In addition, the different terminations of the line only affect the channel response for distances longer than $100 \mathrm{~m}$, which are nearest to the termination, while the effect of the termination is negligible on the first three channels, corresponding to distances of $25 \mathrm{~m}, 50 \mathrm{~m}$ and $75 \mathrm{~m}$. Therefore, considering that a repeater needs to be used every $75 \mathrm{~m}$, the termination of the cable can be completely ignored for the communication purpose.

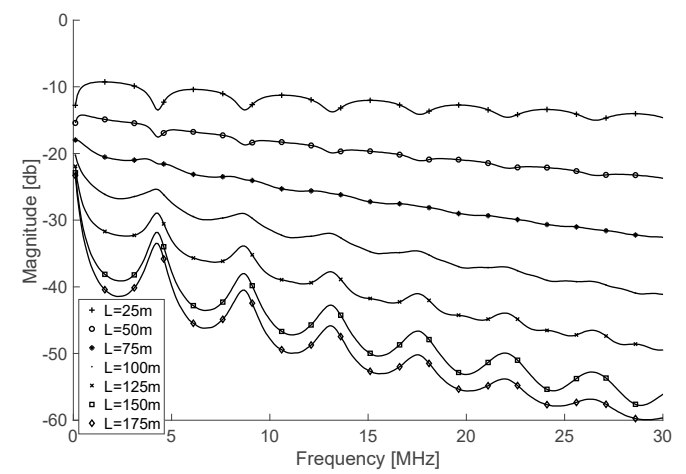

Figure 9. Transfer function magnitudes between different hauled stocks, characteristic impedance termination.

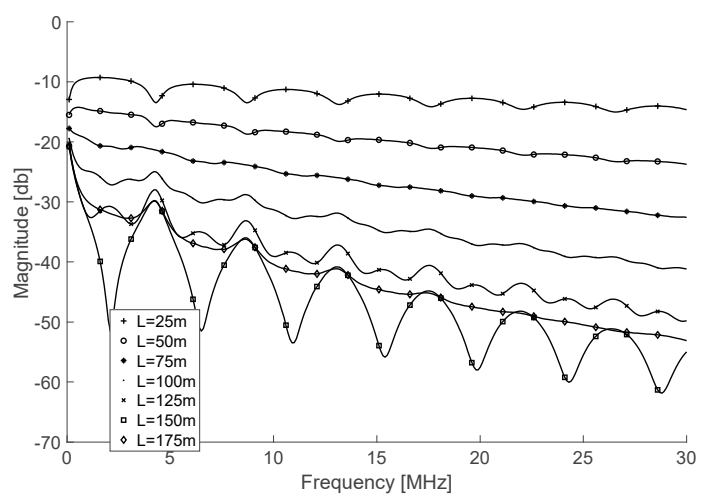

Figure 10. Transfer function magnitudes between different hauled stocks, open circuit termination.

Figures 11 and 12 show the crosstalk relating to conductor 7 (with 6 as common ground) when conductor 5 is excited, with the terminations of conductor 7 being set as the characteristic impedance 
in Figure 11, and open circuit in Figure 12. Considering the magnitudes shown in the above figures, it is evident that for distances longer than $50 \mathrm{~m}$ the presence of the crosstalk signal is highly attenuated (more than $40 \mathrm{db}$ in most frequency bands), whereas for the shortest distance of $25 \mathrm{~m}$, the crosstalk attenuation less than $15 \mathrm{db}$, which is still acceptable taking into account the magnitudes of the signals described previously. As for the frequency responses, the effect of the termination on the crosstalk attenuation is significant only for distances longer than $100 \mathrm{~m}$.

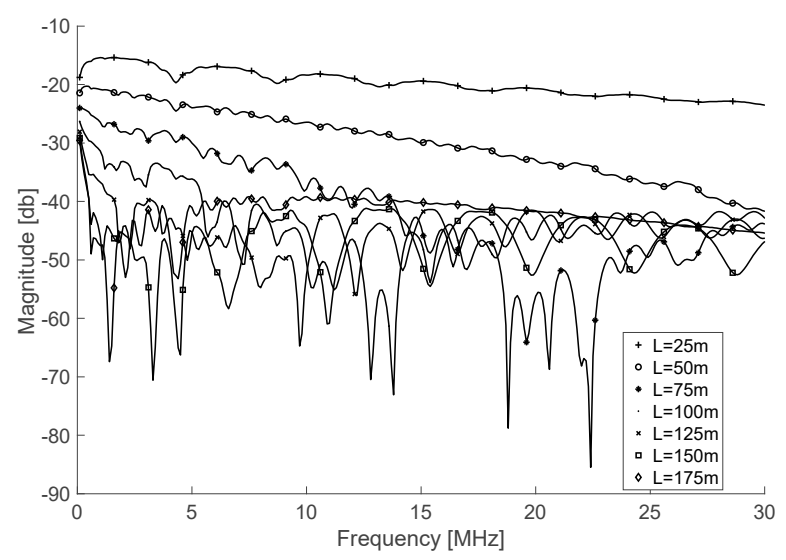

Figure 11. Crosstalk as a function of distance and frequency, characteristic impedance termination.

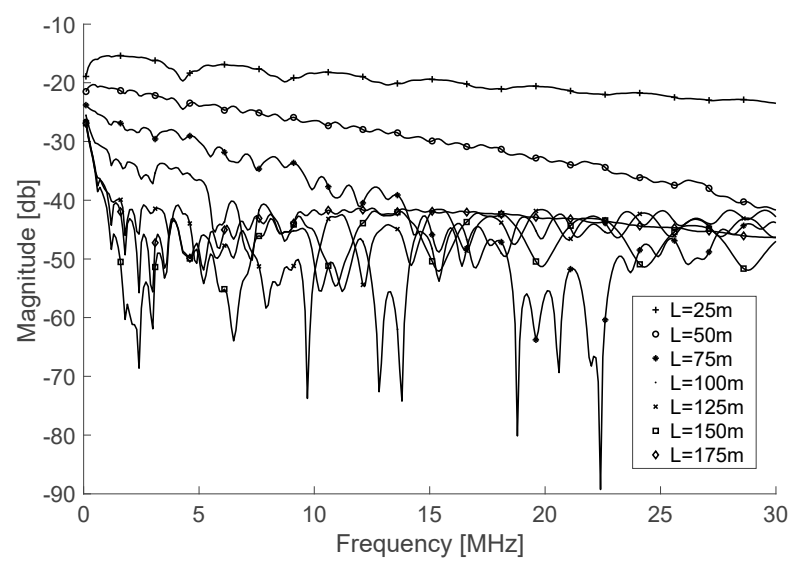

Figure 12. Crosstalk as a function of distance and frequency, open circuit termination.

\subsection{Channel Capacity}

By using the Shannon-Hartley theorem, we calculated the theoretical capacities of the seven channels whose frequency responses are shown in Figure 7. The channel capacity is expressed as a function of the signal-to-noise power ratio $S / N$, where $S$ and $N$ represent, respectively, the power of the transmitted signal and the power of an additive white Gaussian noise at the receiver. If we consider both $S$ and $N$ independent from frequency, then the power of the received signal is given by $|H(f)|^{2} S$, where $H(f)$ denotes the frequency response of the channel, and the capacity of the channel may be written as:

$$
C\left(\frac{S}{N}\right)=\int_{B_{\min }}^{B_{\max }} \log _{2}\left(1+|H(f)|^{2} \frac{S}{N}\right) d f,
$$

where the capacity is expressed in bits per second.

A numerical calculation of (6) was performed using a trapezoidal interpolation, and considering $B_{\min }=2 \mathrm{MHz}$ and $B_{\max }=30 \mathrm{MHz}$, that is, the bandwidth limits of the HOMEPLUG AV protocol that uses OFDM. We note that the performance of the OFDM modulation method has been reported to be, in general, very close to the limit given by the theoretical channel capacity as calculated in (6). 
We considered a transmission power of $-55 \mathrm{dbm} / \mathrm{Hz}$ over the entire frequency band, which is typical for PLC devices, and a background white Gaussian noise with power spectral density between $-115 \mathrm{dbm} / \mathrm{Hz}$, which represents a typical low-noise scenario, and $-80 \mathrm{dbm} / \mathrm{Hz}$, which represents a highly noisy channel. This corresponds to signal-to-noise power ratios (SNRs) at the transmitter between $25 \mathrm{db}$ and $60 \mathrm{db}$, which at the receiver imply per-carrier SNRs between $-35 \mathrm{db}$ and $50 \mathrm{db}$, considering the frequency responses previously obtained.

Figure 13 shows the resulting channel capacities for increasing distance from the transmitter, considering signal-to-noise power ratio (SNR) values from 25 to $60 \mathrm{db}$.

Considering a highly noisy scenario of SNR $=25 \mathrm{db}$, the capacity of the shortest-path channel $(25 \mathrm{~m})$ is about $65 \mathrm{Mbit} / \mathrm{s}$, and the capacity of the longer-path channel $(175 \mathrm{~m})$ is $1.6 \mathrm{Mbit} / \mathrm{s}$. In a low-noise scenario (SNR $=60 \mathrm{db}$ ), the capacities increase up to $220 \mathrm{Mbit} / \mathrm{s}$ for the short path and 57 $\mathrm{Mbit} / \mathrm{s}$ for the long path. Moving from one coach to the successive one, the gain loss is on average 10 $\mathrm{db}$. In addition, for shorter distances, the channel is adequate for a high-speed broadband transmission, for example for video broadcasting and infotainment applications. To better characterize the channel, many performance indicators were calculated, and a bit error rate analysis was carried out, as shown in the following sections.

It is worth noticing that the transmission power of $-55 \mathrm{dBm} / \mathrm{Hz}$ used above is taken as reference value, but this value should be analyzed with respect to railway system EMC constraints; in addition, the real noise levels on UIC cables under regular operations should be measured in order to evaluate the actual operating point of Figure 13.

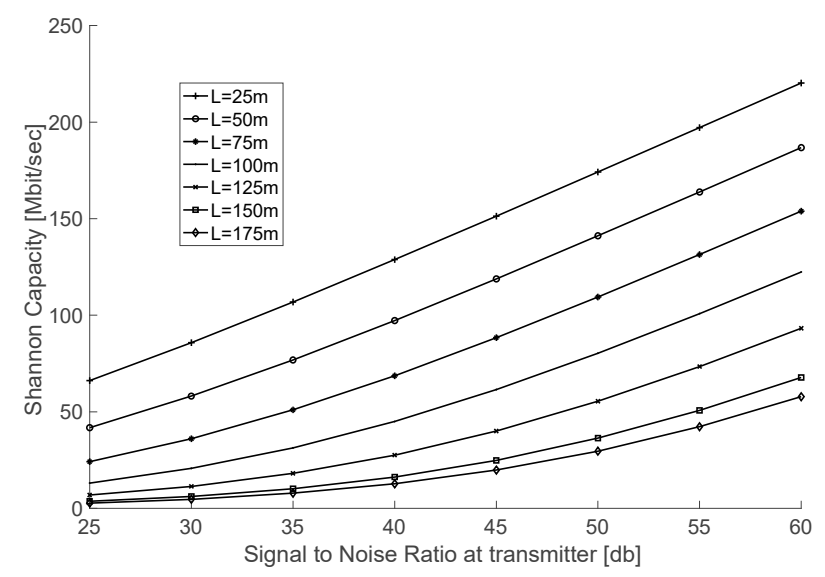

Figure 13. Theoretical channel capacities as a function of the signal to noise power ratio.

\subsection{Root-Mean-Square Delay Spread and Cumulative Density Function}

In most used communication protocols, as for example the OFDM, a guard interval is placed between successive symbols, so that the intersymbol interference is eliminated and full equalization is achieved. The drawback of this technique is that the presence of the guard interval reduces the transmission efficiency.

A different option (to reduce the loss in transmission efficiency) is to set the guard interval to approximately three-times the root-mean-square delay spread (RMSDS) of the channel [20] (the square root of the second central moment of the power-delay profile). This design choice does not totally eliminate intersymbol interference: it reduces the transmission efficiency loss but causes some performance degradation.

Reducing carrier spacing, that is, increasing the number of carriers for the same bandwidth, would have the effect of increasing transmission: in this approach, the transmission efficiency loss is compensated by increased complexity of the transmission protocol. in [20].

In the following Table 1, the RMSDS has been calculated according to the definition reported 
Table 1. RMS Delay Spread.

\begin{tabular}{ccc}
\hline Termination & Characteristic Impedance & Open Circuit \\
\hline$L=25 \mathrm{~m}$ & $5.2117 \times 10^{-8} \mathrm{~s}$ & $5.2131 \times 10^{-8} \mathrm{~s}$ \\
$L=50 \mathrm{~m}$ & $3.1263 \times 10^{-8} \mathrm{~s}$ & $2.9852 \times 10^{-8} \mathrm{~s}$ \\
$L=75 \mathrm{~m}$ & $5.4672 \times 10^{-8} \mathrm{~s}$ & $6.2786 \times 10^{-8} \mathrm{~s}$ \\
$L=100 \mathrm{~m}$ & $1.5101 \times 10^{-7} \mathrm{~s}$ & $1.8992 \times 10^{-7} \mathrm{~s}$ \\
$L=125 \mathrm{~m}$ & $3.1154 \times 10^{-7} \mathrm{~s}$ & $3.9555 \times 10^{-7} \mathrm{~s}$ \\
$L=150 \mathrm{~m}$ & $5.1555 \times 10^{-7} \mathrm{~s}$ & $5.0826 \times 10^{-7} \mathrm{~s}$ \\
$L=175 \mathrm{~m}$ & $6.0195 \times 10^{-7} \mathrm{~s}$ & $4.1073 \times 10^{-7} \mathrm{~s}$ \\
\hline
\end{tabular}

The RMSDS values reported in Table 2 represent important information for the choice of the guard interval duration. As previously observed for frequency responses and crosstalk attenuations, the RMSDS values do not differ significantly for distances less than $75 \mathrm{~m}$, and the need of a repeater every $75 \mathrm{~m}$ has been underlined. As a result, a guard interval of $150 \mathrm{~ns}$ would be sufficient for the transmission, and considering that the typical OFDM symbol duration for broadband PLC is $40 \mu \mathrm{s}$, the efficiency reduction due to the use of the guard interval is of the order of $0.4 \%$.

To evaluate the channel performances, the cumulative density function (CDF) relating to each channel for configurations has been calculated. The CDF is defined as follows, and it gives information on how much bandwidth, in the chosen frequency range, is below a certain amount of attenuation:

$$
\operatorname{CDF}(G)=\frac{\left|\left\{f:\left|S_{21}(F)\right|<G\right\}\right|}{|\{f\}|} .
$$

The results are shown in Figure 14 for the case of termination on the characteristic impedance. For the shortest path channel of $25 \mathrm{~m}$, the magnitude of the frequency response is larger than $-20 \mathrm{db}$ for $100 \%$ of the frequency range, while for the channel of $75 \mathrm{~m}, 65 \%$ of the frequency range is below $-30 \mathrm{db}$.

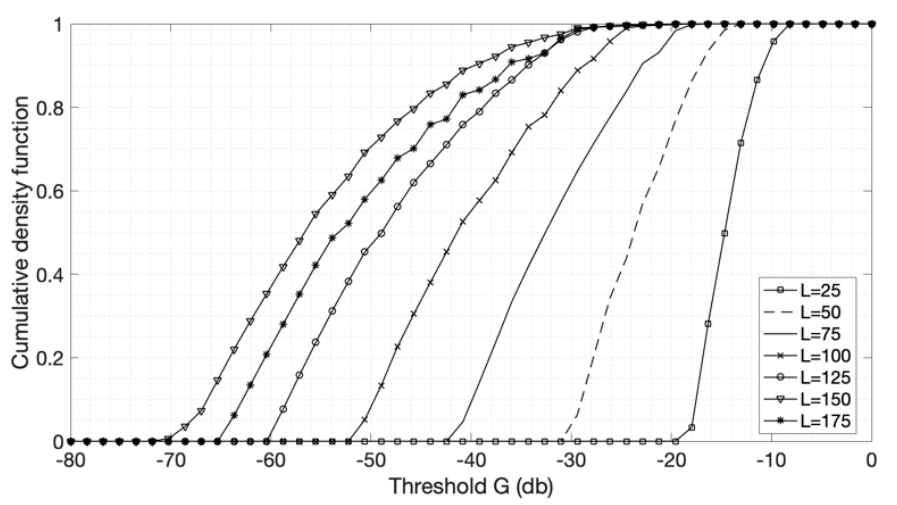

Figure 14. Cumulative density function (CDF) relating to each channel.

\subsection{Bit Error Rate}

The bit error rate of a QPSK-OFDM transmission was calculated by the authors, as a function of the signal-to-noise ratio at the transmitter and distance between the modems. We considered 900 carriers between 2 and $30 \mathrm{MHz}$, and we calculated the BER of each carrier corresponding to the SNR at the receiver after the attenuation of the channel response previously determined. Then, we evaluated an average BER of the channel over the whole frequency band, and the results are shown in Table 2 and Figure 15. 


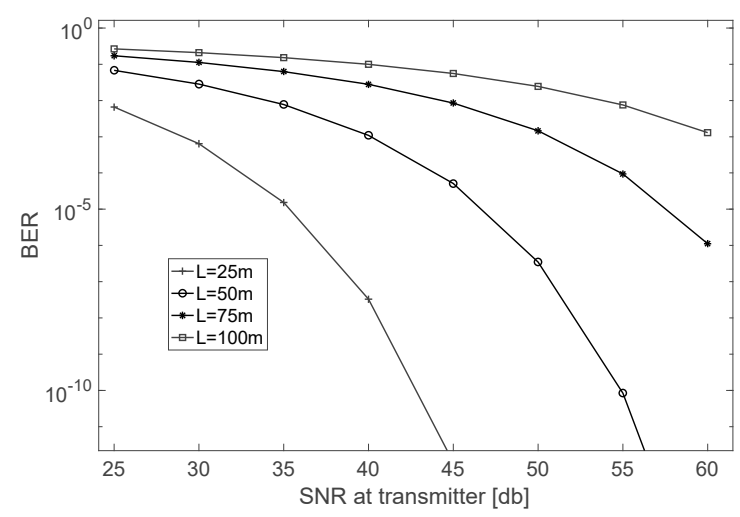

Figure 15. Bit error rate as a function of SNR at the transmitter and distance.

Table 2. Bit error rate as a function of SNR at the transmitter and distance.

\begin{tabular}{|c|c|c|c|c|c|c|c|c|}
\hline SNR (TX) & $25 \mathrm{db}$ & $30 \mathrm{db}$ & $35 \mathrm{db}$ & $40 \mathrm{db}$ & $45 \mathrm{db}$ & $50 \mathrm{db}$ & $55 \mathrm{db}$ & $60 \mathrm{db}$ \\
\hline$L=25 \mathrm{~m}$ & $6.59 \times 10^{-3}$ & $6.42 \times 10^{-4}$ & $1.53 \times 10^{-5}$ & $3.28 \times 10^{-8}$ & $1.05 \times 10^{-12}$ & 0 & 0 & 0 \\
\hline$L=50 \mathrm{~m}$ & $6.84 \times 10^{-2}$ & $2.84 \times 10^{-2}$ & $7.78 \times 10^{-3}$ & $1.10 \times 10^{-3}$ & $5.10 \times 10^{-5}$ & $3.50 \times 10^{-7}$ & $8.45 \times 10^{-11}$ & $5.43 \times 10^{-17}$ \\
\hline$L=75 \mathrm{~m}$ & $1.72 \times 10^{-1}$ & $1.13 \times 10^{-1}$ & $6.31 \times 10^{-2}$ & $2.78 \times 10^{-2}$ & $8.53 \times 10^{-3}$ & $1.46 \times 10^{-3}$ & $9.35 \times 10^{-5}$ & $1.12 \times 10^{-6}$ \\
\hline$L=100 \mathrm{~m}$ & $2.66 \times 10^{-1}$ & $2.10 \times 10^{-1}$ & $1.53 \times 10^{-1}$ & $9.95 \times 10^{-2}$ & $5.55 \times 10^{-2}$ & $2.45 \times 10^{-2}$ & $7.55 \times 10^{-3}$ & $1.29 \times 10^{-3}$ \\
\hline$L=125 \mathrm{~m}$ & $3.36 \times 10^{-1}$ & $2.90 \times 10^{-1}$ & $2.39 \times 10^{-1}$ & $1.84 \times 10^{-1}$ & $1.31 \times 10^{-1}$ & $8.25 \times 10^{-2}$ & $4.40 \times 10^{-2}$ & $1.81 \times 10^{-2}$ \\
\hline$L=150 \mathrm{~m}$ & $3.84 \times 10^{-1}$ & $3.49 \times 10^{-1}$ & $3.08 \times 10^{-1}$ & $2.60 \times 10^{-1}$ & $2.08 \times 10^{-1}$ & $1.55 \times 10^{-1}$ & $1.05 \times 10^{-1}$ & $6.20 \times 10^{-2}$ \\
\hline$L=175 \mathrm{~m}$ & $4.01 \times 10^{-1}$ & $3.71 \times 10^{-1}$ & $3.34 \times 10^{-1}$ & $2.90 \times 10^{-1}$ & $2.41 \times 10^{-1}$ & $1.89 \times 10^{-1}$ & $1.38 \times 10^{-1}$ & $9.01 \times 10^{-2}$ \\
\hline
\end{tabular}

For a data transmission system, a reliability threshold is usually taken as BER $=1 \times 10^{-3}$; higher BER means a less reliable transmission that might lead to unacceptable performances. It can be observed that for a reliable transmission, that is, BER values lower than $1 \times 10^{-3}$, the signal can cover $75 \mathrm{~m}$ in the less noisy condition of $60 \mathrm{db}$ SNR, and less than $25 \mathrm{~m}$ in the worst scenario of $25 \mathrm{db}$ SNR. Also, a gain loss of about $10 \mathrm{db}$ every $25 \mathrm{~m}$ can be observed from Table 2, in order to maintain a fixed BER level.

\section{Conclusions}

In this paper, the authors investigate the use of UIC cables, already present onboard most trains, to send high-speed data to be used as additional services for passengers or security/safety setups. This study shows that, in old trains, it would be possible to include new services that need high-speed data communication without the need for installing additional cable harnesses. These services can be related, for instance, to infotainment for passengers, onboard safety and so on.

The analysis is carried out taking into account all fundamental functions of the UIC cables in order to guarantee that the relative signals are not disrupted by the additional data transfer required to implement a broadband power line transmission. The results show that the signal integrity of pre-existing data is kept, and that a high-speed connection is achievable using the multicarrier modulation schemes typical of the PLC broadband modems. The analysis assumes certain noise levels (that are not measured) and gives guidelines for the correct design of the system. In particular, the results reported in this work give a detailed characterization of the multiconductor cable as a communication medium in the $\mathrm{MHz}$ frequency range, and show that to guarantee a high quality of communication, the use of a repeater every three hauled stocks is needed.

Author Contributions: Conceptualization, methodology, software, validation, formal analysis, writing S.B. and M.T., supervision, F.R.

Funding: This research received no external funding.

Conflicts of Interest: The authors declare no conflict of interest. 


\section{References}

1. Van Rensburg, P.A.J.; Ferreira, H.C.; Snyders, A.J. An experimental setup for in-circuit optimization of broadband automotive power-line communications. In Proceedings of the IEEE International Symposium on Power Line Communications and Its Applications 2005, Vancouver, BC, Canada, 6-8 April 2005; pp. 322-325.

2. Barmada, S.; Raugi, M.; Tucci, M.; Maryanka, Y.; Amrani, O. PLC systems for electric vehicles and smart grid applications. In Proceedings of the IEEE International Symposium on Power Line Communications and Its Applications 2013, Johannesburg, South Africa, 24-27 March 2013; pp. 23-28.

3. Barmada, S.; Raugi, M.; Tucci, M.; Zheng, T. Power line communication in a full electric vehicle: Measurements, modelling and analysis. In Proceedings of the IEEE International Symposium on Power Line Communications and Its Applications 2010, Rio de Janeiro, Brazil, March 2010; pp. 331-336.

4. Huck, T.; Schirmer, J.; Hogenm, T.; Dostert, K. Tutorial about the implementation of a vehicular high speed communication system. In Proceedings of the IEEE International Symposium on Power Line Communications and Its Applications 2005, Vancouver, BC, Canada, 6-8 April 2005; pp. 162-166.

5. Degardin, V.; Laly, P.; Lienard, M.; Degauque, P. Impulsive noise on in-vehicle power lines: Characterization and impact on communication performance. In Proceedings of the IEEE International Symposium on Power Line Communications and Its Applications 2006, Orlando, FL, USA, 26-29 March 2006; pp. 222-226.

6. Degardin, V.; Laly, P.; Lienard, M.; Degauque, P. Performances of the HomePlug PHY Layer in the Context of in-vehicle Powerline Communications. In Proceedings of the IEEE International Symposium on Power Line Communications and Its Applications 2007, Pisa, Italy, 26-28 March 2007; pp. 93-98.

7. Liu, E.; Gao, Y.; Samdani, G.; Mukhtar, O.; Korhonen, T. Powerline Communication over Special Systems. In Proceedings of the IEEE International Symposium on Power Line Communications and Its Applications 2005, Vancouver, BC, Canada, 6-8 April 2005; pp. 167-171.

8. Pittolo, A.; De Piante, M.; Versolatto, F.; Tonello, A.M. In-Vehicle Power Line Communication: Differences and Similarities Among the In-Car and the In-Ship Scenarios. IEEE Veh. Technol. Mag. 2016, 11, 43-51. [CrossRef]

9. Tsuzuki, S.; Yoshida, M.; Yamada, Y.; Kawasaki, H.; Murai, K.; Matsuyama, K.; Suzuki, M. Characteristics of Power-Line Channels in Cargo Ships. In Proceedings of the IEEE International Symposium on Power Line Communications and Its Applications 2007, Pisa, Italy, 26-28 March 2007. [CrossRef]

10. Barmada, S.; Bellanti, L.; Raugi, M.; Tucci, M. Analysis of power-line communication channels in ships. IEEE Trans. Veh. Technol. 2010, 59, 3161-3170. [CrossRef]

11. Antoniali, M.; Tonello, A.M.; Lenardon, M.; Qualizza, A. Measurements and analysis of PLC channels in a cruise ship. In Proceedings of the IEEE International Symposium on Power Line Communications and Its Applications 2011, Udine, Italy, 3-6 April 2011; pp. 102-107.

12. Jones, C.H. Communication over aircraft power lines. In Proceedings of the IEEE International Symposium on Power Line Communications and Its Applications 2006, Orlando, FL, USA, 26-29 March 2006; pp. $149-154$.

13. Degardin, V.; Junqua, I.; Lienard, M.; Degauque, P.; Bertuol, S. Theoretical approach to the feasibility of power-line communication in aircrafts. IEEE Trans. Veh. Technol. 2013, 62, 1362-1366. [CrossRef]

14. UIC 558. Ligne de Telecommande et D'information. Caracteristiquest Techniques Unifiees por L'equipment des Voitures RIC; Union Internationale des Chemins de fer: Paris, France, 1992.

15. Barmada, S.; Gaggelli, A.; Masini, P.; Musolino, A.; Rizzo, R.; Tucci, M. Modeling of UIC Cables in railway Systems for their Use as Power Line Communications Channels. Appl. Comput. Electromagn. J. 2009, 24, 609-617.

16. Barmada, S.; Tucci, M.; Romano, F. Channel Capacity Evaluation of UIC Cables for High Speed Data Transmission Onboard Regional Trains. In Proceedings of the Third International Conference on Railway Technology: Research, Development and Maintenance, Cagliari, Italy, 5-8 April 2016; pp. 1-12.

17. Barmada, S.; Gaggelli, A.; Musolino, A.; Rizzo, R.; Raugi, M.; Tucci, M. Design of a PLC System Onboard Trains: Selection and Analysis of the PLC Channel. In Proceedings of the IEEE International Symposium on Power Line Communications and Its Applications 2008, Jeju Island, Korea, 2-4 April 2008; pp. 13-17.

18. Paul, C. Analysis of Multiconductor Transmission Lines; Wiley: Hoboken, NJ, USA, 1994. 
19. Eisenstadt, E.R.; Eo, Y. S-parameter-based IC interconnect transmission line characterization. IEEE Trans. Compon. Hybridsmanuf. Technol. 1992, 15, 483-490. [CrossRef]

20. Galli, S. A simplified model for the indoor power line channel. In Proceedings of the IEEE International Symposium on Power Line Communications and Its Applications 2009, Dresden, Germany, 29 March-1 April 2009; pp. 13-19. 